\title{
Simultaneous photometric and spectral analysis of new outburst of V1686 Cyg
}

\author{
H. R. Andreasyan ${ }^{1 *}$, T. Yu. Magakian ${ }^{1}$, T. A. Movsessian ${ }^{1}$, \\ M. H. Gevorgyan ${ }^{1}$ \\ ${ }^{1}$ NAS RA V. Ambartsumian Byurakan Astrophysical Observatory (BAO), Armenia \\ *E-mail: hasmik.andreasyan@gmail.com
}

\begin{abstract}
We present an analysis of the optical observations of Herbig AeBe star V1686 Cyg, which is demonstrating photometric variability up to 3 to 4 magnitudes. We observed this star as a part of our project of young eruptive stars investigation. Observations were performed on $2.6 \mathrm{~m}$ telescope of Byurakan Observatory. In the course of observations we noticed that this star underwent a new outburst. After data reduction we found that full rise and decline of V1686 Cyg brightness for almost 3 magnitudes lasted about 2 months. We were able to trace the changes of the stellar spectrum during the outburst. They are discussed and compared with other young eruptive variables.
\end{abstract}

Keywords: stars - variables - T Tauri - Herbig Ae/Be - photometry spectroscopy.

\section{Introduction}

$\mathrm{LkH} \alpha 224$ is Herbig AeBe star with characteristic $\mathrm{H} \alpha$ emission. It is located in the vicinity of bright BD+404124 star, which is showing very strong $\mathrm{H} \alpha$ line in emission. For the first time this star was mentioned by Herbig (1960), when he studied AeBe stars connected with bright nebulosities. There are other emission line stars near this object, e.g. V1318 Cyg, so they create a small cluster of young stars. Optically brightest member of this cluster is BD+404124, which itself is Herbig Be star. Shevchenko et al (1991) estimate a distance to this group as $980 \mathrm{pc}$. In general this region is embedded in the dense molecular cloud. The most complete information about photometric behavior of $\mathrm{LkH} \alpha 224=\mathrm{V} 1686 \mathrm{Cyg}$ so far is collected in the work of Shevchenko et al. (1991). Based on their observations, the star demonstrates irregular variability: 2 mag outbursts of four-five days duration, with about 2 months of minimum brightness between. Beside 
these short-term variations, this star shows also long-term variations: it decreased in brightness by more that $4.5 \mathrm{mag}$ in $7.5 \mathrm{yr}$, and then raised its brightness by $4 \mathrm{mag}$ in $4 \mathrm{yr}$. In the survey of Herbst \& Shevchenko (1999) of UBVR photometry of Herbig AeBe stars, carried out since 1983, they estimate V=14.07 for V1686 Cyg mean brightness, while Hillenbrand et al. (1995) found out $V=16.13$. Concerning the spectrum of this star, it should be noted that no final agreement on its spectral type exists. The prominent variations of absorption features make classification process very problematic. Hillenbrand et al. (1995) suggest spectral type B5Ve, while Mora et al. (2001) estimated the spectral type to be A4, but with more than 5 subclasses error. Hernandez et al. (2004) obtained spectra of all Herbig AeBe stars from the Herbig-Bell Catalog (Herbig \& Bell 1988) and noticed that spectrum of V1686 Cyg looks very different compared to the previous observations, and classified it as F9. In the work of Magakian et al. (1997) the spectrum of V1686 Cyg was studied in details. They confirmed that this star is an early-type star, where only the broad $\mathrm{H} \alpha$ emission with an asymmetric profile is visible in the optical range. There are no detailed studies of this star during its brightening phase, so this work will for the first time show the differences in the spectrum of V1686 Cyg in two phases.

\section{Observations}

In 2015 we started our project on investigation of eruptive stars. Observations were carried out on $2.6 \mathrm{~m}$ telescope in Byurakan Observatory. We used SCORPIO spectral camera, and obtained direct images, as well as long-slit spectra. As an object of our previous interests we started our observations for V1318 Cyg young variable star, and at the same time obtained data for the neighbor V1686 Cyg star. Observations were implemented from September 2015 to July 2017. Data reduction was done in the usual way, using IRAF and ESO-MIDAS programs. Photometric estimations were done by aperture photometry. For calibration we used several stars in the field, measured by Hillenbrand et al. (1995) and Shevchenko et al. (1991). The typical errors for our data are about $0^{m} .02-0^{m} .03$. We also obtained spectra for this star from Sept. 2015 to Dec. 2016, with different spectral resolutions: $0.50,0.80,1.50$ and $2.65 \AA /$ pix.

\section{The new outburst of V1686 Cyg}

In the period of Sept. 2015 - Aug. 2016 V1686 Cyg did not show any significant variability. But in Aug. 2016, during our regular observation we noticed that V1686 Cyg raised its brightness. In Fig. 1 we see the appearance of the star before the outburst, while in Fig. 2 it is already raised its brightness. For the comparison we found out the images of IPHAS 


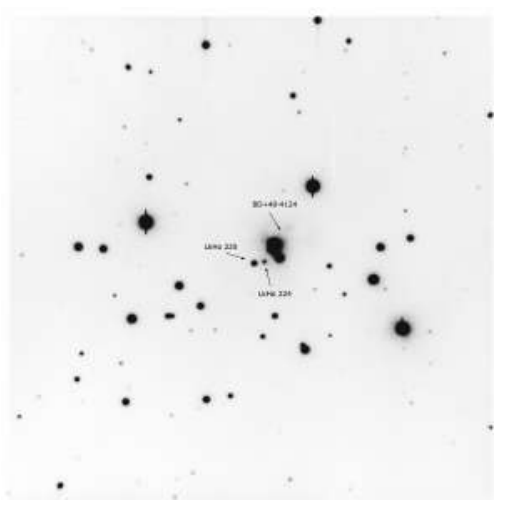

Figure 1: Before the outburst

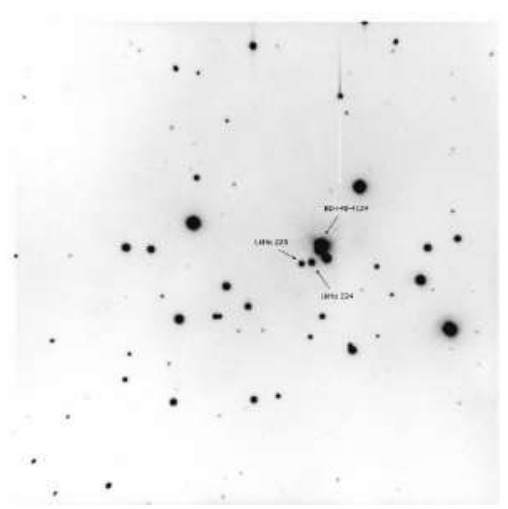

Figure 2: During the outburst

survey in R, I bands, and estimated the brightness of the star in 20032006. The continuation of our observations demonstrated that V1686 Cyg star raised its brightness for almost 3 magnitudes for 2-3 months, and then again gradually came to its typical appearance in minimum. In the Table 1 and Table 2 we present the results of our photometry of $2.6 \mathrm{~m}$ telescope images and IPHAS survey images correspondingly. Such type of brightness fluctuations is usual for this star and is in a good agreement with the previous works, described above.

\begin{tabular}{|c|c|c|c|c|}
\hline Date & B & V & R & I \\
\hline 22.09 .2015 & 17.22 & 15.88 & 14.68 & 13.27 \\
\hline 18.11 .2015 & 17.18 & 15.96 & 14.87 & 13.56 \\
\hline 31.03 .2016 & 17.03 & 15.61 & 14.48 & 13.07 \\
\hline 10.06 .2016 & 17.42 & 16.05 & 14.76 & 13.00 \\
\hline 23.08 .2016 & 14.82 & 13.50 & 12.48 & 11.58 \\
\hline 20.12 .2016 & 16.34 & 14.98 & 13.62 & 12.49 \\
\hline 17.07 .2017 & 16.99 & 15.66 & 14.63 & 13.53 \\
\hline
\end{tabular}

Table 1: V1686 Cyg photometry

\begin{tabular}{|c|c|c|}
\hline Year & R & I \\
\hline 2003 & 13.29 & 13.37 \\
\hline 2004 & 15.00 & 13.90 \\
\hline 2006 & 14.98 & 13.79 \\
\hline
\end{tabular}

Table 2: Photometry of IPHAS images of V1686 Cyg star 


\section{Spectrum of V1686 Cyg in minimum}

As was pointed out above, we obtained the spectra of V1686 Cyg during the whole period of its outburst. First spectra, taken before the outburst, are quite typical for this star, and very similar to description of Magakian \& Movsessian (1997). One can see broad $\mathrm{H} \alpha$ in emission, and [OI] and [SII] forbidden lines, which belong to background nebulosity around the star. After the ending of outburst, in Nov. 2016, spectrum looks the same way, without any significant differences.

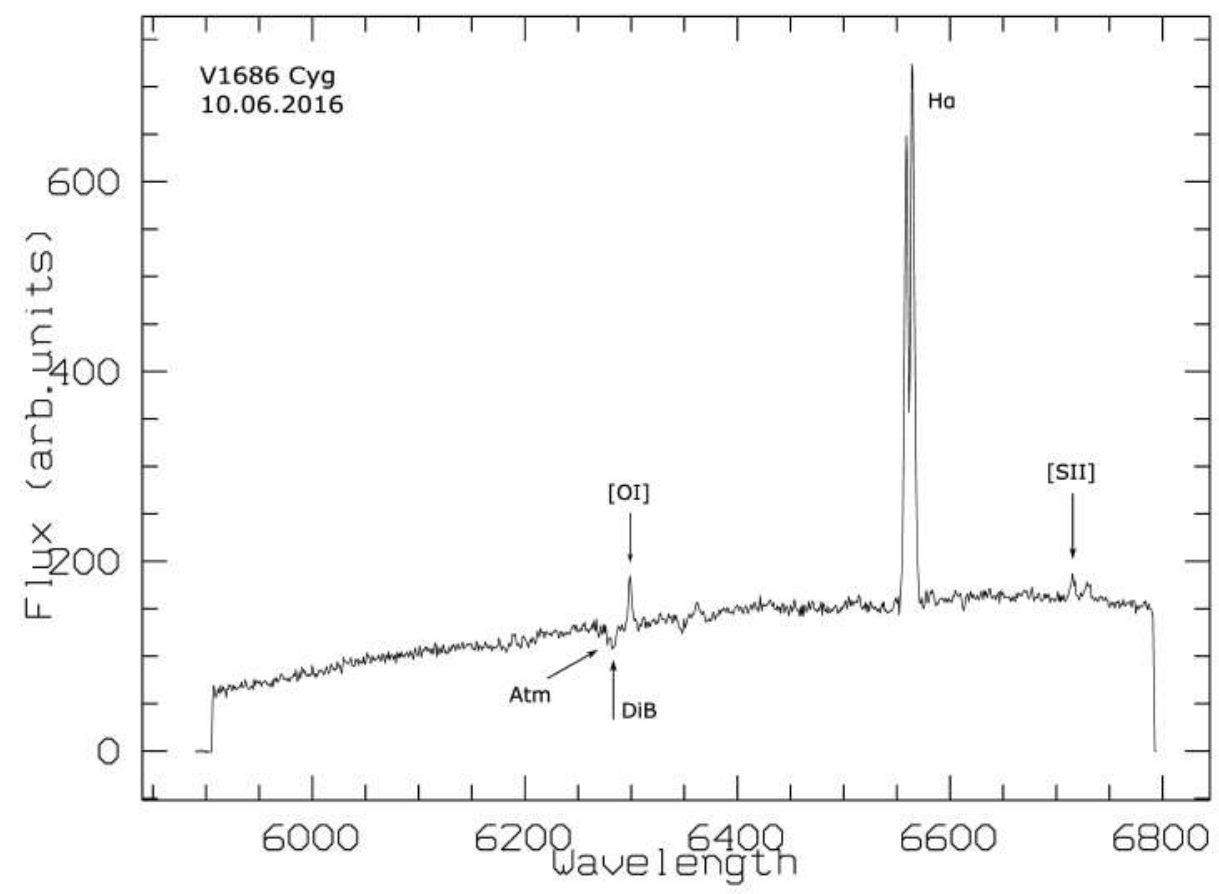

Figure 3: Spectrum of $\mathrm{LkH} \alpha 224$ in minimum

\section{Spectrum of V1686 Cyg in maximum}

Spectrum of Aug. 24 of 2016 corresponds to the outburst of V1686 Cyg. It keeps its appearance at least until Aug. 30 of 2016. We do not know exactly when the star started lowering its brightness. The next spectrum we obtained in Nov. 6, when this star returned to its mean brightness, and it does not differ from the spectra before the outburst. One can see that during the outburst V1686 Cyg spectrum is quite different and has remarkable changes especially in the profile of $\mathrm{H} \alpha$ line: it has narrow, but very deep, lowering down the continuum absorption component, superposed on the bright emission. Besides, in maximum one can notice a variety of 
low-excitation FeI and FeII emission lines.

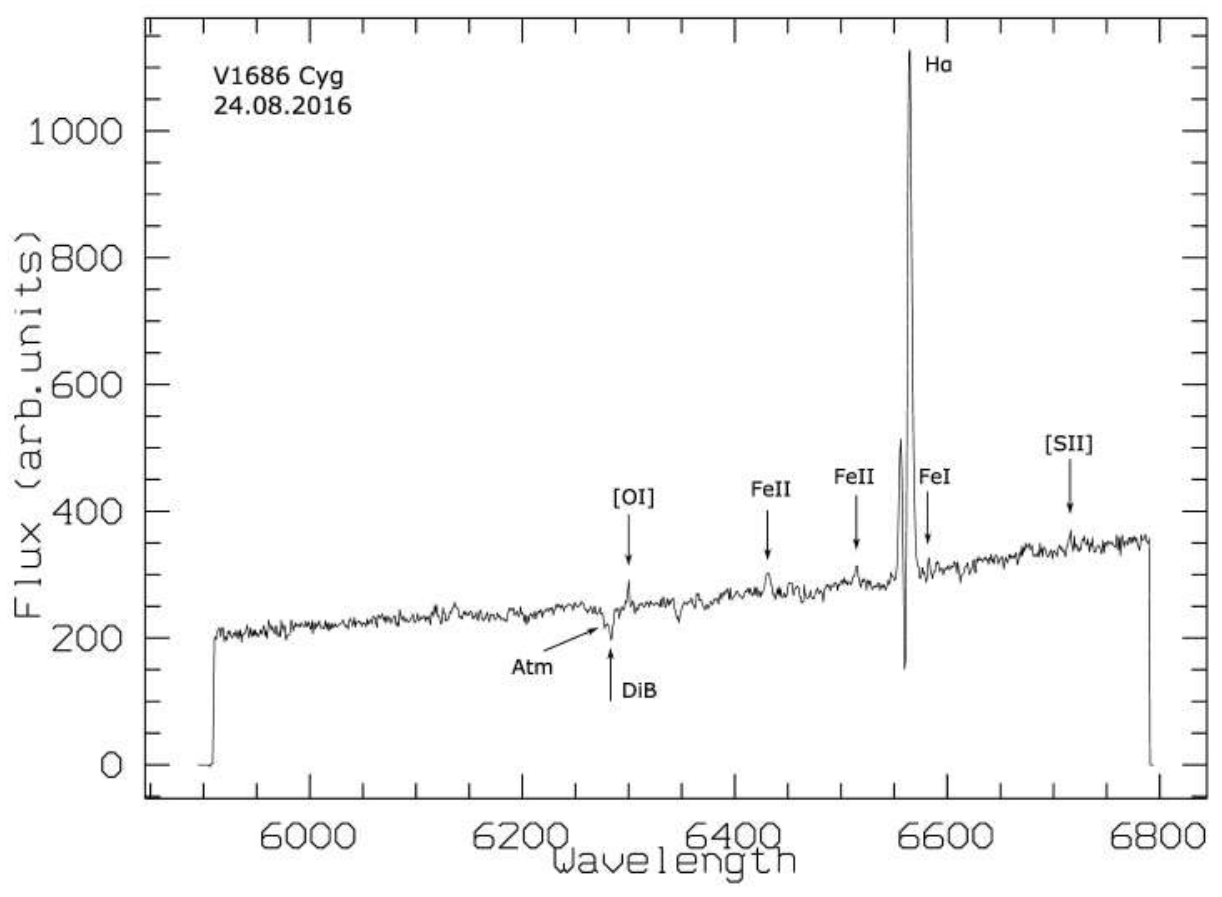

Figure 4: Spectrum of $\mathrm{LkH} \alpha 224$ during the outburst

Besides, it is ought to mention that in both minimum and maximum spectra we detected diffuse interstellar band (DIB) $6284 \AA$, which is distinguished from the nearby atmospheric absorption line $6278 \AA$. This DIB is among the strongest ones (Herbig 1995).

\section{Summary}

The new photometric and spectroscopic data confirm, that V1686 Cyg is HAeBe type star, which constantly vary its brightness for 2-3 magnitudes, with maxima about 1-2 months duration. Spectral type of this star could be early A.

The prominent changes in its spectrum in maximal and quiescent states, observed in detail for the first time, unambiguously show that such outbursts are accompanied with significant increase of mass loss from the star. From the $\mathrm{H} \alpha$ line profile changes and appearance of iron emission lines we can conclude that during the outburst the star became surrounded by an enlarging envelope which afterwards dissipates into space.

We should also note, that this star is immersed in dense molecular core, so $\lambda 6284 \AA$ DIB line which is detected in both minimum and maximum spectra of V1868 Cyg, is probably related with this cloud. 


\section{References}

Herbig, G. H. 1960, ApJ 4, 337

Herbig, G. H. 1995, A\&A, 33, 19

Herbig, G. H., \& Bell, K. R. 1988, Third catalog of emission-line stars of the Orion population., cels.book, 90p.

Herbst, W., \& Shevchenko, V. S., 1999, AJ 118, 1043

Hernández, J., Calvet, N., Briceño, C., Hartmann, L., \& Berlind, P. 2004, A\&A, 127, 1682

Hillenbrand, L. A.; Meyer, M. R.; Strom, S. E.; Skrutskie, M. F. 1995, AJ 109, 280

Shevchenko, V. S. 1991, SvA 35, 229

Magakyan, T. Y., \& Movsesyan, T. A. 1997, AstL, 23, 666

Mora, A.; Merín, B.; Solano, E.; et al. 2001, A\&A 378, 116 